\title{
Downregulation of BRAF-activated non-coding RNA suppresses the proliferation, migration and invasion, and induces apoptosis of hepatocellular carcinoma cells
}

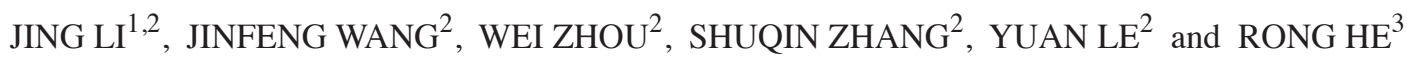 \\ ${ }^{1}$ Department of Pediatrics, Shengjing Hospital of China Medical University, Shenyang, Liaoning 110004; \\ ${ }^{2}$ Department of Pediatrics, The First Affiliated Hospital of Liaoning Medical University, Jinzhou, Liaoning 121001; \\ ${ }^{3}$ Department of Clinical Genetics, Shengjing Hospital of China Medical University, Shenyang, Liaoning 110004, P.R. China
}

Received April 1, 2016; Accepted July 3, 2017

DOI: $10.3892 / 01.2017 .6770$

\begin{abstract}
The long non-coding RNA BRAF-activated non-coding RNA (BANCR) has been reported to serve essential roles in the progress of a various cancer types. The purpose of the present study was to investigate the effect of IncRNA BANCR in HCC cells. The expression of BANCR in the HCC cell line Huh7 and a normal liver cell line were determined using reverse transcription-quantitative polymerase chain reaction. The expression of BANCR in Huh7 cells was downregulated by short hairpin (sh)RNA. Subsequently, the proliferation, apoptosis, migration and invasion rates were determined, along with the activity of MAPK/ERK kinase (MEK) and mitogen-activated protein kinase signaling pathways. The results revealed that BANCR was overexpressed in Huh7 cells when compared with normal liver cells. The downregulation of BANCR significantly inhibited the proliferation and colony formation ability, and induced cell cycle arrest and apoptosis of Huh7 cells. The migration and invasion of Huh7 cells were also suppressed in BANCR shRNA-transfected cells. The downregulation of BANCR significantly inhibited the activity of MEK, extracellular signal-regulated kinase and janus kinase signaling pathways. Collectively, these findings demonstrated that the lncRNA BANCR is oncogenic in Huh7 cells, and may be a promising molecular target for HCC therapy.
\end{abstract}

\section{Introduction}

Hepatocellular carcinoma (HCC) is one of the most common types of malignancy and is the third leading cause of cancer-associated mortality worldwide (1). There are

Correspondence to: Dr Rong He, Department of Clinical Genetics, Shengjing Hospital of China Medical University, 36 Sanhao Street, Shenyang, Liaoning 110004, P.R. China

E-mail: rong_he0110@sina.com

Key words: hepatocellular carcinoma cell, BRAF-activated non-coding RNA, proliferation, apoptosis, migration, invasion $\sim 500,000$ cases diagnosed as HCC each year, which represents $>5 \%$ of all cancer cases (2). The incidence of HCC has a considerable geographic variation, with the majority of the cases occurring in developing countries, including Southeast Asia and sub-Saharan Africa (3). However, in recent decades, epidemiological studies have also indicated a rising trend in the incidence and mortality of HCC in Western countries (4). Despite substantial and accelerated studies focused on the treatment of HCC, the five-year survival rate of advanced HCC remains poor (5-7). Thus, the development of novel and efficient therapy strategies for HCC are warranted.

Long non-coding RNA (lncRNA) is a group of noncoding RNAs that are $>200$ nucleotides in length, and serve regulatory roles in different physiological processes, including growth, differentiation, senescence and apoptosis (8). Recently, increasing evidence suggest that the dysregulation of lncRNAs are involved in diverse pathological conditions, in particular in various types of cancer (9-11). BRAF-activated non-coding RNA (BANCR), a 693-bp lncRNA on chromosome 9, was first identified to be overexpressed in melanoma cells and serve as a regulator in the migration of melanoma cells $(12,13)$. Reportedly, BANCR is abnormally expressed in gastric tumor (14), papillary thyroid carcinoma (15), colorectal cancer (16), retinoblastoma (17), papillary thyroid carcinoma (18) and non-small cell lung cancer (19). Despite the majority of these studies indicating an oncogenic property of BANCR, Sun et al (19) and Shi et al (16) reported tumor suppressive activity of BANCR in non-small cell lung cancer, and colorectal carcinoma, respectively.

In the present study, the expression of BANCR in Huh7 cells was downregulated using short hairpin (sh)RNA, and the effect of BANCR on proliferation, apoptosis, migration and invasion of HCC cells was investigated in vitro. Furthermore, the underlying mechanisms of BANCR in HCC were explored.

\section{Materials and methods}

Cell lines, culture condition and treatment. The human hepatocellular carcinoma cell line Huh7 was purchased from the Type Culture Collection of the Chinese Academy of Sciences (Shanghai, China). Human normal liver L-02 cells were 
purchased from Zhongqiaoxinzhou Biotechnology Co., Ltd (Shanghai, China). Cells were maintained in Dulbecco's modified Eagle's medium or RPMI-1640 (both from Gibco; Thermo Fisher Scientific, Inc., Waltham, MA, USA) and supplemented with $10 \%$ fetal bovine serum (Hyclone; GE Healthcare Life Sciences, Logan, UT, USA) in a humidified atmosphere of $5 \% \mathrm{CO}_{2}$ at $37^{\circ} \mathrm{C}$. Cells was transfected with BANCR shRNA or negative control (NC) shRNA using Lipofectamine ${ }^{\mathrm{TM}} 2000$ (Invitrogen; Thermo Fisher Scientific, Inc.) according to the manufacturer's protocol. The target sequences were as follows: BANCR shRNA, 5'-GGAGTGGCGACTATAGCAA-3' and NC shRNA, 5'-TTCTCCGAACGTGTCACGT-3'.

Reverse transcription-quantitative polymerase chain reaction $(P C R)$. Total RNA was extracted using the RNApure total RNA extraction kit and reverse transcribed to cDNA using Super M-MLV reverse transcriptase at $25^{\circ} \mathrm{C}$ for $10 \mathrm{~min}$ followed by $42^{\circ} \mathrm{C}$ for $50 \mathrm{~min}$. (both from Bioteke Corporation, Beijing, China). SYBR Green-based PCR was performed using an Exicycler $^{\mathrm{TM}} 96$ real-time PCR system (Bioneer Corporation, Daejeon, Korea) with 2X Power Taq PCR Master mix (Bioteke Corporation), the thermocycling conditions was as follows: $95^{\circ} \mathrm{C}$ for $10 \mathrm{~min}$ followed by 40 cycles of $95^{\circ} \mathrm{C}$ for $10 \mathrm{sec}$, $60^{\circ} \mathrm{C}$ for $20 \mathrm{sec}$ and $72^{\circ} \mathrm{C}$ for $30 \mathrm{sec}$. The specific primers for BANCR were as follows: Forward, 5'-TCAGAAGAAACA AGAGGGAGG-3' and reverse, 5'-AGCAGCATGAACTGG GAAAC-3'. The specific primers of $\beta$-actin were as follows: Forward, 5'-CTTAGTTGCGTTACACCCTTTCTTG-3' and reverse, 5'-CTGTCACCTTCACCGTTCCAGTTT-3'. Each experiment was repeated for three times. The relative BANCR mRNA level was calculated using the $2^{-\Delta \Delta C q}$ method (20) and normalized to $\beta$-actin expression.

Western blot analysis. Protein from each group was extracted using the Whole Cell Lysis kit (Wanleibio, Shenyang, China). A total of $40 \mu \mathrm{g}$ protein per lane were electrophoresed using 8,10 or $13 \%$ SDS-PAGE and subsequently electrotransfered to polyvinylidene fluoride membranes (EMD Millipore, Billerica, MA, USA). After blocking in 5\% non-fat milk at room temperature for $1 \mathrm{~h}$, the membranes were incubated at $4^{\circ} \mathrm{C}$ overnight with the following primary antibodies: $\mathrm{Bcl}-2$ (1:400 dilution, cat. no. BA0412), Bax (1:400 dilution, cat. no. BA0315) (both from Wuhan Boster Biological Technology, Ltd., Wuhan, China), cleaved caspase-3 (1:1,000 dilution; cat. no. ab2302, Abcam, Cambridge, MA, USA), phosphorylated (p)-extracellular signal-regulated kinase (p-ERK; 1:500 dilution; cat. no. bs-1522R), ERK (1:500 dilution; cat. no. bs-2637R), p-janus kinase (p-JNK; 1:500 dilution; cat. no. bs-1640R), JNK (1:500 dilution; cat. no. bs-10562R), p-P38 (1:500 dilution; cat. no. bs-5477R), P38 (1:500 dilution; cat. no. bs-0637R) (all from BIOSS, Beijing, China), p-MAPK/ERK kinase (p-MEK; 1:200 dilution; cat. no. sc-271914), MEK (1:200 dilution; cat. no. sc-219), and $\beta$-actin (1:1,000 dilution; cat. no. sc-47778) (all from Santa Cruz Biotechnology, Inc., Dallas, TX, USA). Subsequently, the membranes were incubated with horseradish peroxidase-conjugated goat anti rabbit (cat. no. WLA023) or goat anti mouse (cat. no. WLA024) secondary antibodies (Wanleibio) at $37^{\circ} \mathrm{C}$ for $45 \mathrm{~min}$. The specific bands were visualized with the Immu-Plus ECL system (Wanleibio), and the protein levels were semi-quantified using densitometry analysis that performed by Gel-Pro Analyzer Version 3.0 (Media Cybernetics, Silver Spring, MD, USA). $\beta$-actin served as an internal control.

Cell viability assay. Cells were seeded in a 96-well plate at a density of $3 \times 10^{3}$ cells/well and cultivated at $37^{\circ} \mathrm{C}$ for $24 \mathrm{~h}$. Subsequently, cells were transfected with BANCR shRNA or NC shRNA, and further incubated for 24, 48, 72 or 96 h. Cells were then incubated with $0.5 \mathrm{mg} / \mathrm{ml} \mathrm{MTT}$ for $4 \mathrm{~h}$, followed by the addition of $200 \mu \mathrm{l}$ dimethyl sulfoxide. Lastly, the absorbance at $490 \mathrm{~nm}$ was measured using the ELX-800 microplate spectrophotometer (Biotek Instruments, Inc., Winooski, VT, USA). For colony formation, cells were seeded in a $35-\mathrm{mm}$ culture dish (300 cells/dish), and maintained in culture media supplemented with $10 \%$ fetal bovine serum for two weeks. The cell clones were fixed with $4 \%$ paraformaldehyde at room temperature for $20 \mathrm{~min}$, then stained with Wright-Giemsa dye at room temperature for $5 \mathrm{~min}$. Colony formation rate was calculated as follows: (colony number/seeding number) x100\%.

Flow cytometry. Cells were collected $48 \mathrm{~h}$ post-transfection for cell cycle analysis, cells were incubated with $25 \mu \mathrm{l}$ propidium iodide (Beyotime Institute of Biotechnology, Haimen, China) for $30 \mathrm{~min}$ at $37^{\circ} \mathrm{C}$ in the dark. For apoptosis detection, cells were incubated with $5 \mu \mathrm{l}$ Annexin V-FITC and $5 \mu 1$ propidium iodide following the manufacturer's protocol (Nanjing KeyGen Biotech Co., Ltd., Nanjing, China). Following staining, the cell cycle or apoptosis status was analyzed using flow cytometry (BD Accuri C6; BD Biosciences, Franklin Lakes, NJ, USA).

Hoechst staining. Cells were seeded on 12-well plates at a density of $5 \times 10^{4}$ cells/well, transfected with BANCR shRNA or NC shRNA. A total of $48 \mathrm{~h}$ after transfection, cells were fixed and stained with $2 \mu \mathrm{g} / \mathrm{ml}$ Hoeschst staining solution (Beyotime Institute of Biotechnology) according to the manufacturer's protocol. Apoptotic nuclei were observed under a fluorescent microscope (BX53; Olympus Corporation, Tokyo, Japan).

Wound healing assay. Cells were allowed to grow until $80-90 \%$ confluence was achieved on 6-well plates, then transfected with BANCR shRNA or NC shRNA. A total of $48 \mathrm{~h}$ after transfection, cells were incubated with $1 \mu \mathrm{g} / \mathrm{ml}$ mitomycin C (Sigma-Aldrich; Merck KGaA, Darmstadt, Germany) for $1 \mathrm{~h}$, and a wound was created with a $200-\mu 1$ pipette tip. Subsequently, cells were cultured in a humidified atmosphere of $5 \% \mathrm{CO}_{2}$ at $37^{\circ} \mathrm{C}$ for $24 \mathrm{~h}$. The migration distance was measured under an inverted phase contrast microscope (AE31; Motic Instruments, Richmond, BC, Canada).

Transwell assays. Transwell chambers (Corning Incorporated, Corning, NY, USA) were pre-coated with $40 \mu 1$ Matrigel (BD Biosciences) and placed on to 24-well plates. Cells from each group were seeded in the top chamber, $2 \times 10^{4}$ cells/well. A total of $800 \mu \mathrm{l}$ cell culture medium supplemented with $20 \%$ fetal bovine serum was added to the lower chamber as a chemoattractant. The non-invading cells on the upper-side of the membrane were removed with cotton swabs 24 after incubation. The invasive cells were fixed with $4 \%$ paraformaldehyde at room temperature for $20 \mathrm{~min}$ and stained with $0.5 \%$ crystal 

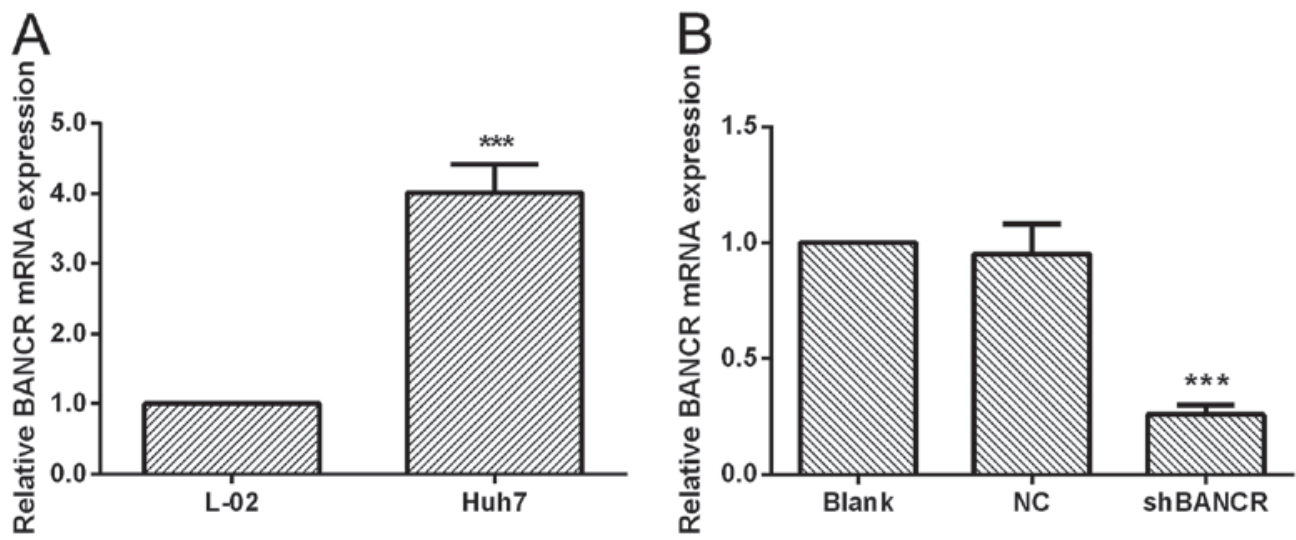

Figure 1. IncRNA BANCR is overexpressed in Huh7 cells compared with normal liver cells. (A) The expression of BANCR in Huh7 hepatocellular carcinoma cells and normal liver cells were determined by reverse transcription-quantitative polymerase chain reaction. (B) Transfection of BANCR shRNA significantly decreased the level of BANCR in Huh7 cells. Data are expressed as the mean \pm standard deviation $(\mathrm{n}=3) .{ }^{* * *} \mathrm{P}<0.001$ compared with $\mathrm{L}-02$ cells or NC shRNA-transfected cells. IncRNA, long non-coding RNA; BANCR, BRAF-activated non-coding RNA; NC, negative control; shRNA/sh, short hairpin RNA.

violet solution at room temperature for $5 \mathrm{~min}$. The number of invasive cells was counted under inverted phase-contrast microscope in a blinded manner.

Statistical analysis. The cell viability assay was repeated five times; all other experiments were repeated three times. All values are expressed as the mean \pm standard deviation. Differences between groups were analyzed using one-way analysis of variance followed by Bonferroni's multiple comarisons test with SPSS 16.0 software (SPSS, Inc., Chicago, IL, USA). $\mathrm{P}<0.05$ were considered to indicate a statistically significant difference.

\section{Results}

BANCR is overexpressed in HCC cells. To assess the biological role of BANCR in the progression of $\mathrm{HCC}$, the expression level of BANCR in Huh7 HCC cells and normal liver cells was measured by using RT-PCR. As presented in Fig. 1A, BANCR was significantly overexpressed in the human HCC cell line Huh7 when compared with normal liver cells $(\mathrm{P}<0.001)$. Thus, Huh7 cells were transfected with BANCR shRNA or NC shRNA, the expression level of BANCR in transfected cells was also determined (Fig. 1B). The results revealed that transfection with BANCR shRNA resulted in a significant decrease $(72.59 \pm 1.59 \%)$ in BANCR levels compared with NC shRNA-transfected Huh7 cells $(\mathrm{P}<0.001)$, suggesting that the expression of BANCR was significantly inhibited by BANCR shRNA.

Downregulation of BANCR suppresses the viability of $H C C$ cells. To investigate whether BANCR was functionally involved in HCC cell viability, the proliferation of HCC cells was detected using an MTT assay. The results demonstrated that the proliferation of Huh7 cells transfected with BANCR shRNA were significantly decreased compared with NC shRNA-transfected cells (Fig. 2A; P<0.01). Simultaneously, the clonogenicity tests revealed that downregulation of BANCR resulted in a significantly decreased colony formation rate compared with the NC shRNA group (Fig. 2B; $\mathrm{P}<0.001)$. Furthermore, cell cycle analysis was performed to assess the role of BANCR in the regulation of cell proliferation, as presented in Fig. $2 \mathrm{C}$, the $\mathrm{G}_{0} / \mathrm{G}_{1}$ phase cell proportion was increased significantly in shRNA BANCR-transfected cells compared with NC shRNA-transfected cells $(\mathrm{P}<0.05)$. Thus, the downregulation of BANCR may inhibit HCC cell proliferation by induction of cell cycle arrest.

Downregulation of BANCR induces the apoptosis of HCC cells. The apoptosis status of BANCR shRNA-transfected cells were determined using flow cytometry (Fig. 3A), it was demonstrated that the apoptosis cell proportion in Huh7 cells was significantly higher in BANCR shRNA-transfected cells compared with NC shRNA-transfected cells $(\mathrm{P}<0.001)$, and this was also confirmed by Hoechst staining. As presented in Fig. 3B, typical apoptotic bodies were notably observed in BANCR shRNA-transfected cells. In addition, the expression of apoptosis-associated proteins was measured (Fig. 3C). As expected, when compared with the NC group, downregulation of BANCR significantly inhibited the expression of anti-apoptotic protein Bcl-2, and this was accompanied by an increase in proapoptotic protein Bax and cleaved caspase-3. Taken together, downregulation of BANCR promoted apoptosis of HCC cells.

Downregulation of BANCR inhibits the migration and invasion of HCC cells. To clarify the effect of BANCR on migration and invasion of HCC cells, wound healing and Transwell assays were performed. As presented in Fig. 4A and $\mathrm{B}$, when compared with the $\mathrm{NC}$ group, the migration rate, and invasive cells in the BANCR shRNA-transfected group were significantly decreased $(\mathrm{P}<0.05)$, indicating that downregulation of BANCR can inhibit the migration and invasion of HCC cells.

Downregulation of BANCR inactivates MEK, ERK and $J N K$, but does not affect the activity of P38 MAPK signaling pathway. The activation of MAPK and MEK pathway were examined using western blot analysis, to explore the potential underlying mechanisms of BANCR in the progress of HCC. It was revealed that the MEK, ERK and JNK signaling pathways were inactivated significantly in BANCR shRNA-transfected 

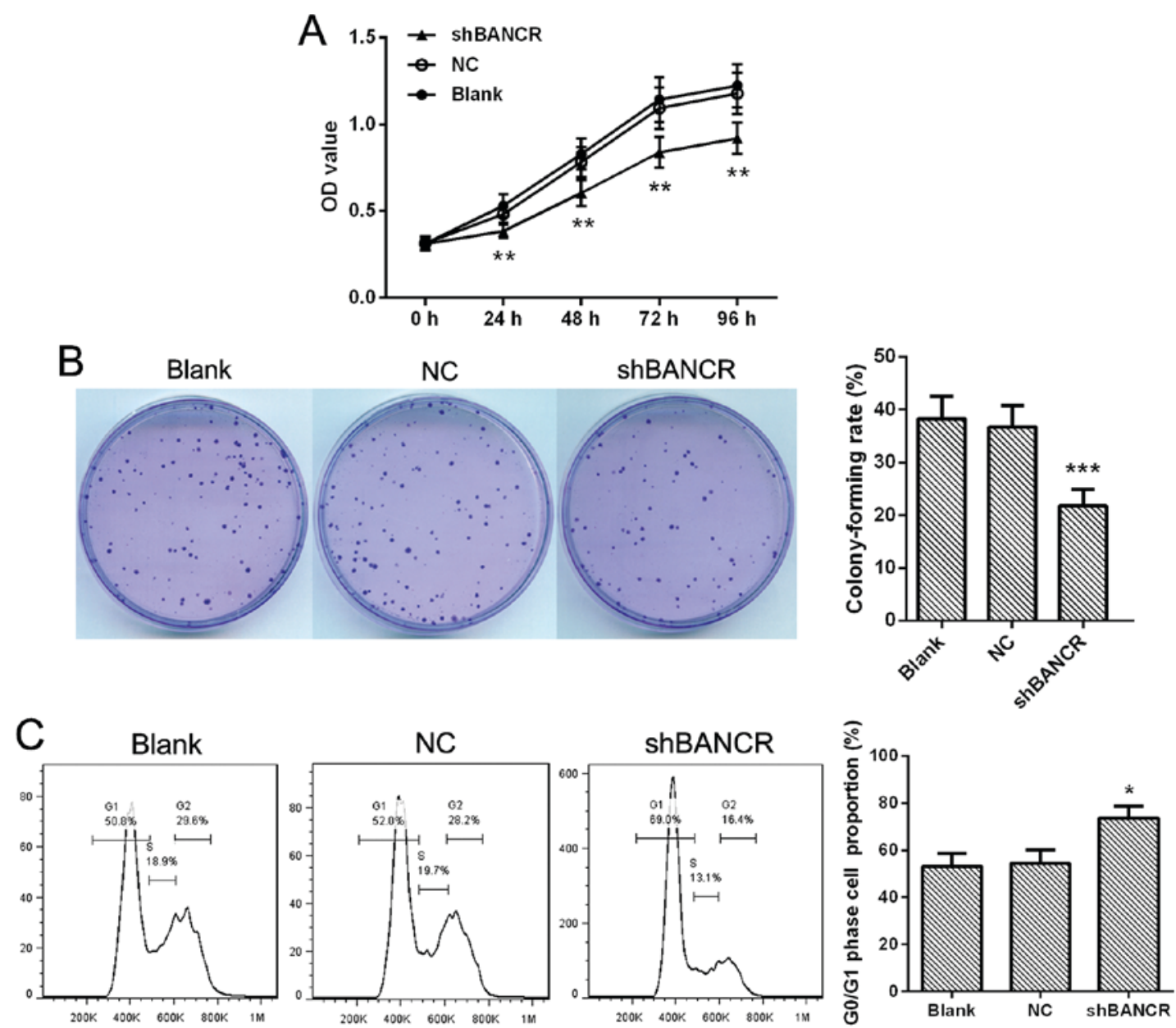

Figure 2. Downregulation of BANCR suppresses the viability of Huh7 cells. (A) Huh7 cells were cultured in 96-well plates, the proliferation at 24, 48, 72 and $96 \mathrm{~h}$ were determined by MTT assay, $\mathrm{n}=5$. (B) Cells were cultured at 35-mm culture dish for two weeks, stained with Wright-Giemsa, and the colony formation rate was calculated, $\mathrm{n}=3$. (C) A total of $48 \mathrm{~h}$ after transfection, the cells were stained with propidium iodide, cell cycle was detected by flow cytometry, $\mathrm{n}=3$. Representative images are presented and data are expressed as the mean \pm standard deviation, ${ }^{*} \mathrm{P}<0.05,{ }^{* *} \mathrm{P}<0.01,{ }^{* * *} \mathrm{P}<0.001$ compared with the $\mathrm{NC}$ group. BANCR, BRAF-activated non-coding RNA; NC, negative control; shRNA/sh, short hairpin RNA.

cells when compared with NC shRNA-transfected cells (Fig. 5A-C). However, the activity of the P38 pathway was not affected by the downregulation of BANCR (Fig. 5D). Thus, these results suggest that the MEK, ERK and JNK signaling pathways were involved in the BANC-associated malignance of HCC cells.

\section{Discussion}

LncRNAs are newly recognized RNAs, which may serve important roles in the development of cancer. Previous studies have reported that the lncRNA BANCR is abnormally expressed in various malignant tumors and participates in tumor development (14-19). In the present study, it was demonstrated that the IncRNA BANCR was overexpressed in the HCC cell line Huh7 when compared with the normal liver cell line L-02. The downregulation of BANCR by shRNA significantly suppressed the proliferative capacity, clonogenicity and induced apoptosis of Huh7 cells. The migratory and invasive ability of Huh7 cells were also inhibited by BANCR shRNA. Furthermore, these tumor inhibitory effects appear to be associated with the repression of MEK, ERK and JNK signaling pathways. These findings demonstrated an essential role for BANCR in the regulation of proliferation, apoptosis, migration and invasion in HCC cells.

BANCR has been identified to be significantly upregulated in human malignant melanoma (21), papillary thyroid carcinoma (18), gastric tumor $(14,22)$ and retinoblastoma tissues (23). Previous studies revealed that the knockdown of BANCR resulted in cell growth inhibition and cell cycle arrest in human thyroid cancer cells (15), and human melanoma cells (21). The aforementioned studies described a tumor promotional role of BANCR. However, other studies have reported that BANCR was expressed at a lower level in various cancer types, and its expression was inversely correlated with tumor malignancy, meanwhile, in vitro experiments on colorectal cancer, non-small cell lung cancer and lung carcinomas cells suggested that BANCR serves as an cancer suppressor gene $(16,19,24)$. Thereby, BANCR serves distinct roles in different tumor types, which may be attributed to tumor heterogenicity. The present study demonstrated that BANCR was significantly overexpressed in Huh7 HCC cells when compared with normal liver cells, and the downregulation of BANCR significantly inhibited proliferation, colony formation and induced cell cycle arrest in Huh7 cells, suggesting a tumor promoter property of BANCR in HCC cells. 

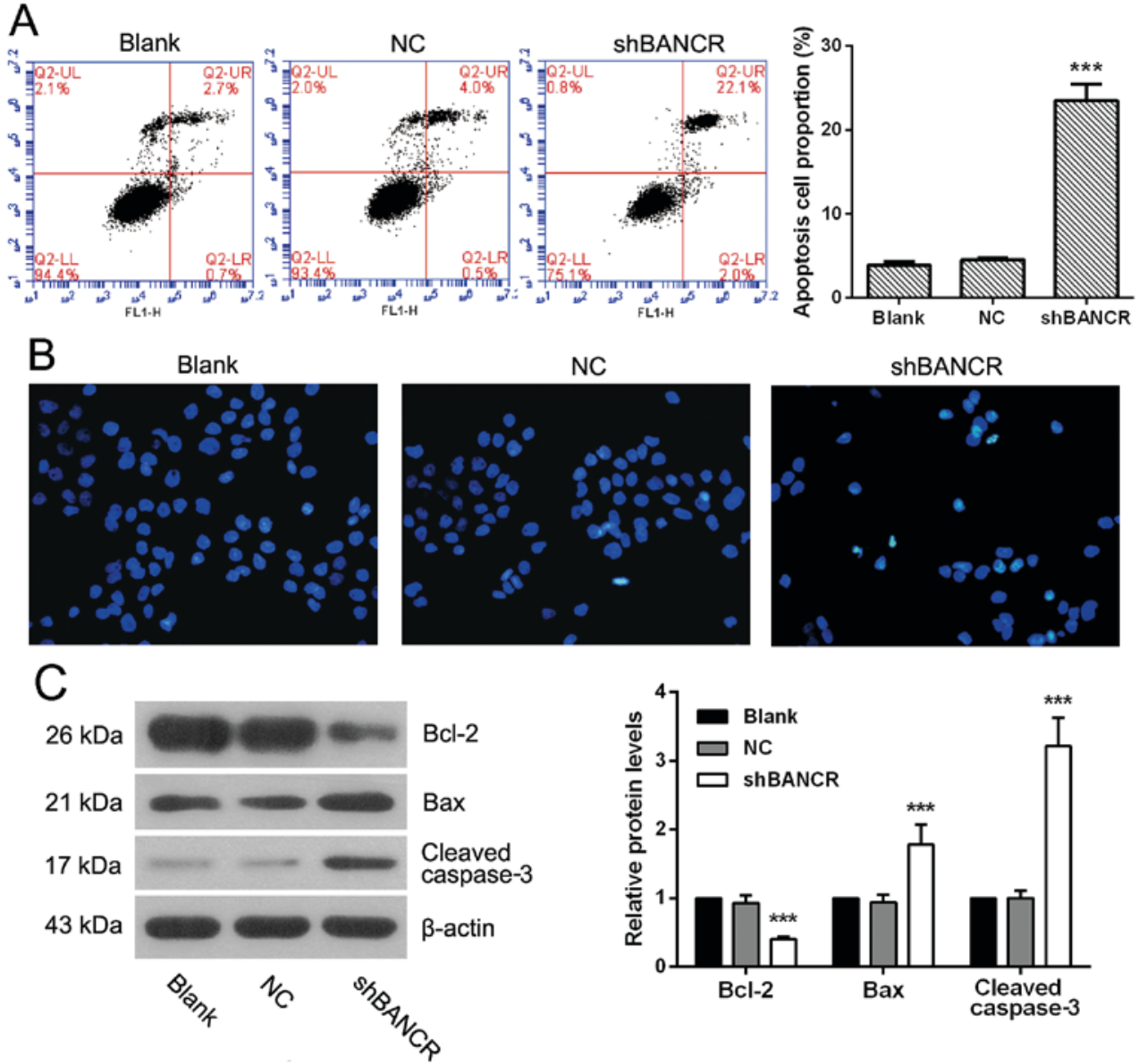

Figure 3. Downregulation of BANCR induces apoptosis of hepatocellular carcinoma cells. (A) Cells were stained with Annexin V-FITC and propidium iodide, apoptosis cells were detected by flow cytometry, $n=3$. (B) Cells were cultured on 12-well plates, stained with Hoeschst solution, apoptosis nucleus were observed under fluorescent microscope, $\mathrm{n}=3$. Scale bar, $50 \mu \mathrm{m}$. (C) The protein level of Bcl-2, Bax and cleaved caspase-3 were determined by western blotting, $\mathrm{n}=3$, representative bands are presented. Data are expressed as mean \pm standard deviation, ${ }^{* * * *} \mathrm{P}<0.001$ compared with the NC group. IncRNA, long non-coding RNA; BANCR, BRAF-activated non-coding RNA; NC, negative control; shRNA, short hairpin RNA.
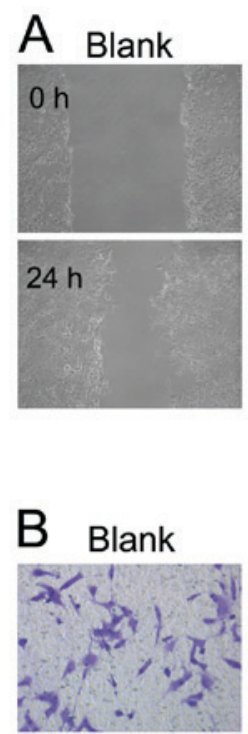

NC
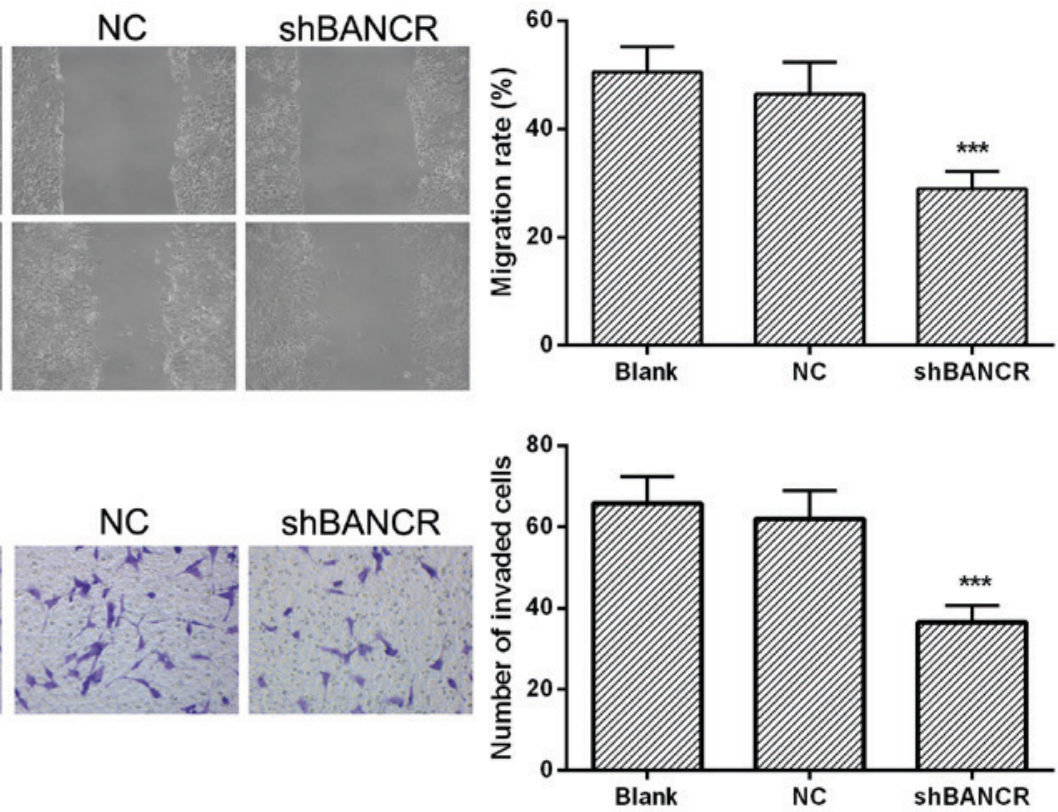

Figure 4. Downregulation of BANCR inhibits the migration and invasion of Huh7 cells. (A) Cells were transfected with BANCR shRNA or NC shRNA, grown to $80-90 \%$ confluence on 6-well plates, wounded with a 200- $\mu$ l pipette tip, and the migration distance at 12 and $24 \mathrm{~h}$ was measured, $\mathrm{n}=3$. Scale bar, $200 \mu \mathrm{m}$. (B) Cells were seeded on Matrigel-coated 24-well plates, the invasiveness of cells was determined using a Transwell assay, $\mathrm{n}=3$. Scale bar, $100 \mu \mathrm{m}$. Representative images are presented and data are expressed as the mean \pm standard deviation, ${ }^{* * *} \mathrm{P}<0.001$ compared with the NC group. lncRNA, long non-coding RNA; BANCR, BRAF-activated non-coding RNA; NC, negative control; shRNA, short hairpin RNA. 
A

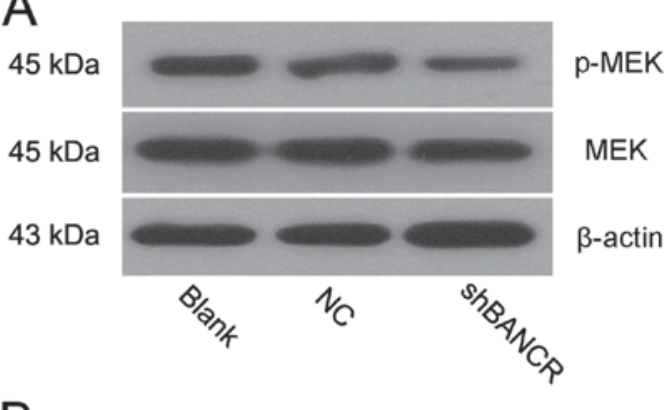

B

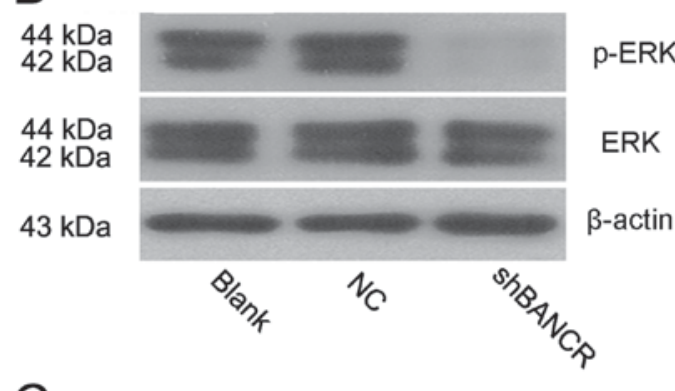

C

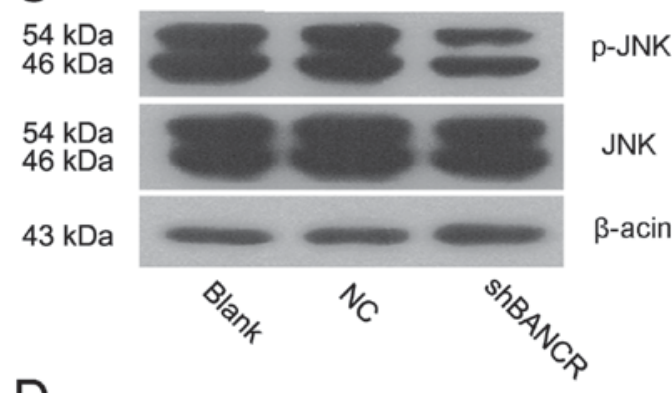

$\mathrm{D}$

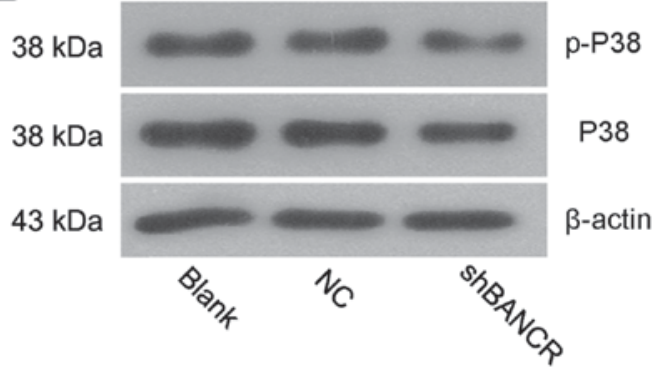

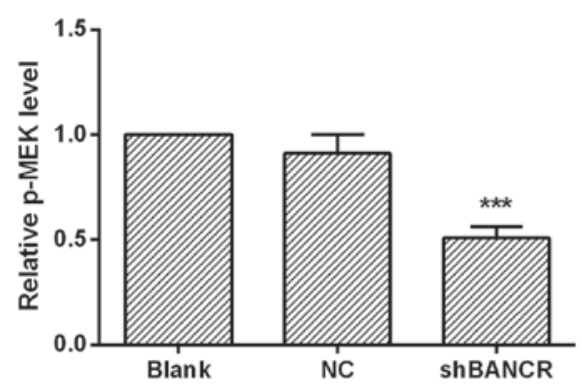
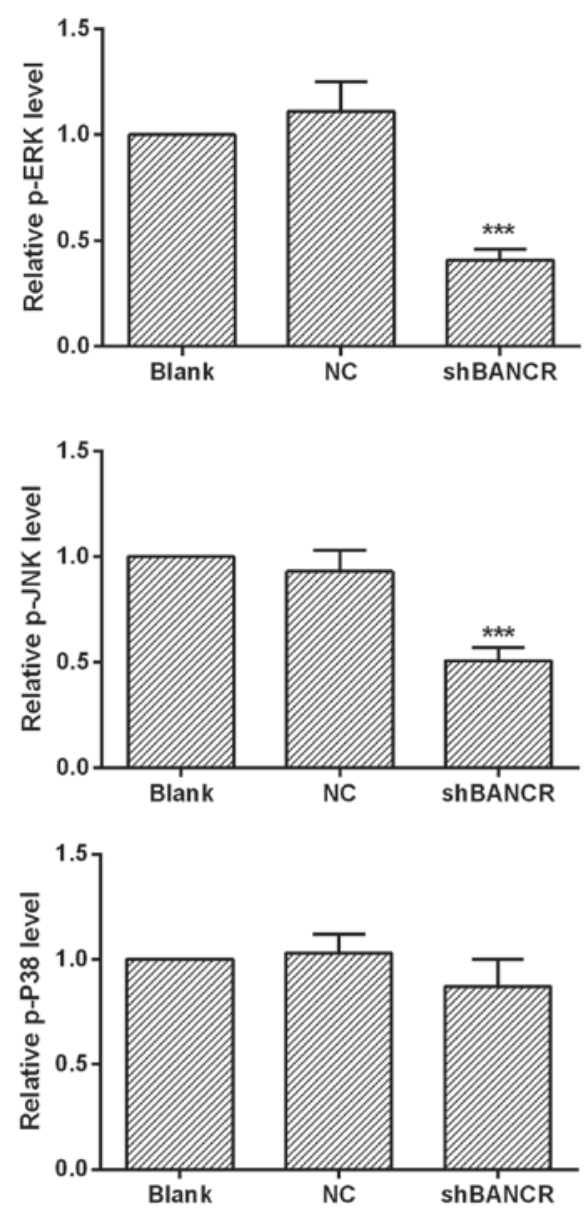

Figure 5. Downregulation of BANCR inactivates MEK, ERK and JNK signaling pathways, but does not affect the p38 mitogen-activated protein kinase pathway. The activity of (A) MEK, (B) ERK, (C) JNK and (D) P38 pathways were determined by western blotting, $n=3$. Representative images are presented and data are expressed as the mean \pm standard deviation, ${ }^{* * *} \mathrm{P}<0.001$ compared with the NC group. lncRNA, long non-coding RNA; BANCR, BRAF-activated non-coding RNA; NC, negative control; shRNA, short hairpin RNA; MEK, MAPK/ERK kinase; ERK, extracellular signal-regulated kinase; JNK, janus kinase.

It was also demonstrated that the repression of BANCR significantly induced apoptosis of Huh7 cells. Given the important roles of apoptosis-associated proteins in the cell apoptosis signaling pathway $(25,26)$, the expression of $\mathrm{Bcl}-2$, Bax and cleaved caspase-3 was determined using western blotting. The results revealed that transfection with BANCR shRNA significantly inhibited the expression of $\mathrm{Bcl}-2$, increased the level of Bax and cleaved caspase-3, further elucidating the role of BANCR in the regulation of apoptosis.

Flockhart et al (12) reported that BANCR was recurrently overexpressed in melanomas tissues, and knockdown of BANCR in melanoma cells significantly reduced cell migration. In addition, Guo et al (27) confirmed that BANCR contributes to the migration of colorectal cancer cells by the promotion of epithelial-mesenchymal transition. In the present study, it was revealed that the migration rate and invasive cell number of Huh7 cells were significantly reduced following BANCR shRNA transfection, these results consistently suggest that the downregulation of BANCR can suppress the migration and invasion of HCC cells.

It is accepted that the abnormal activation of numerous cellular and molecular signaling pathways are involved in the biological process of hepatocarcinogenesis (28-32). MEK and MAPK signaling pathways are key pathways that participate in the regulation of cell proliferation, apoptosis, and differentiation $(33,34)$. The suppression of MEK/ERK and MAPK signaling pathways using chemotherapeutic drugs has yielded major improvements in the management of HCC $(35,36)$. In 
the present study, the downregulation of BANCR significantly inhibited the activity of MEK, ERK and JNK signaling pathways in Huh7 cells, but had no effect on P38 MAPK signaling. Thus, implying that MEK, ERK and JNK pathways may be implicated in BANCR-mediated tumor development, but not the P38 MAPK pathway. These findings are in line with previous studies demonstrating that the activation of MEK and MAPK signaling pathways are positively associated with the expression of BANCR $(21,24,27)$.

Although the downregulation of lncRNA BANCR demonstrated a significant cancer inhibitory effect in the HCC cell line Huh7, the role of lncRNA BANCR on other HCC cell lines remains unclear. A limitation of the present study is that a single hepatocellular carcinoma cell line was used to investigate the effect of lncRNA BANCR. Thus, further studies are warranted to verify the function role of lncRNA BANCR in the development of HCC.

In conclusion, the results of the present study demonstrated that lncRNA BANCR was overexpressed in Huh7 cells when compared with normal liver cells L-02. Downregulation of BANCR significantly inhibited the proliferation, migration, invasion and aggravated apoptosis of Huh7 cells. Parallel to these biological changes, the downregulation of BANCR also suppressed the activity of MEK, ERK and JNK signaling pathways in HCC cells. The present study described a cancer promotion property of BANCR and provided a potential molecular target for the treatment of HCC.

\section{References}

1. Knudsen ES, Gopal P and Singal AG: The changing landscape of hepatocellular carcinoma: Etiology, genetics and therapy. Am J Pathol 184: 574-583, 2014.

2. Ahn J and Flamm SL: Hepatocellular carcinoma. Dis Mon 50: 556-573, 2004.

3. Everson GT: Increasing incidence and pretransplantation screening of hepatocellular carcinoma. Liver Transpl 6 (6 Suppl 2): S2-S10, 2000.

4. El-Serag HB: Epidemiology of viral hepatitis and hepatocellular carcinoma. Gastroenterology 142: 1264-1273.e1, 2012.

5. Bruix J and Llovet JM: Major achievements in hepatocellular carcinoma. Lancet 373: 614-616, 2009.

6. Olsen SK, Brown RS and Siegel AB: Hepatocellular carcinoma: Review of current treatment with a focus on targeted molecular therapies. Therap Adv Gastroenterol 3: 55-66, 2010.

7. Forner A and Bruix J: Biomarkers for early diagnosis of hepatocellular carcinoma. Lancet Oncol 13: 750-751, 2012.

8. Meseure D, Drak Alsibai K, Nicolas A, Bieche I and Morillon A: Long noncoding RNAs as new architects in cancer epigenetics, prognostic biomarkers, and potential therapeutic targets. Biomed Res Int 2015: 320214, 2015.

9. Tsai MC, Spitale RC and Chang HY: Long intergenic noncoding RNAs: New links in cancer progression. Cancer Res 71: 3-7, 2011.

10. Wapinski $\mathrm{O}$ and Chang HY: Long noncoding RNAs and human disease. Trends Cell Biol 21: 354-361, 2011.

11. Spizzo R, Almeida MI, Colombatti A and Calin GA: Long non-coding RNAs and cancer: A new frontier of translational research? Oncogene 31: 4577-4587, 2012.

12. Flockhart RJ, Webster DE, Qu K, Mascarenhas N, Kovalski J, Kretz M and Khavari PA: BRAFV600E remodels the melanocyte transcriptome and induces BANCR to regulate melanoma cell migration. Genome Res 22: 1006-1014, 2012.

13. McCarthy N: Epigenetics. Going places with BANCR. Nat Rev Cancer 12: 451, 2012

14. Zhang ZX, Liu ZQ, Jiang B, Lu XY, Ning XF, Yuan CT and Wang AL: BRAF activated non-coding RNA (BANCR) promoting gastric cancer cells proliferation via regulation of NF- $\kappa$ B1. Biochem Biophys Res Commun 465: 225-231, 2015.
15. Zheng H, Wang M, Jiang L, Chu H, Hu J, Ning J, Li B, Wang D and $\mathrm{Xu}$ J: BRAF-activated long non-coding RNA modulates papillary thyroid carcinoma cell proliferation through regulating thyroid stimulating hormone receptor. Cancer Res Treat 48: 698-707, 2016.

16. Shi Y, Liu Y, Wang J, Jie D, Yun T, Li W, Yan L, Wang K and Feng J: Downregulated long noncoding RNA BANCR promotes the proliferation of colorectal cancer cells via downregulation of p21 expression. PLoS One 10: e0122679, 2015.

17. Su S, Gao J, Wang T, Wang J, Li H and Wang Z: Long non-coding RNA BANCR regulates growth and metastasis and is associated with poor prognosis in retinoblastoma. Tumour Biol 36: 7205-7211, 2015

18. Wang Y, Guo Q, Zhao Y, Chen J, Wang S, Hu J and Sun Y: BRAF-activated long non-coding RNA contributes to cell proliferation and activates autophagy in papillary thyroid carcinoma. Oncol Lett 8: 1947-1952, 2014.

19. Sun M, Liu XH, Wang KM, Nie FQ, Kong R, Yang JS, Xia R, $\mathrm{Xu}$ TP, Jin FY, Liu ZJ, et al: Downregulation of BRAF activated non-coding RNA is associated with poor prognosis for non-small cell lung cancer and promotes metastasis by affecting epithelial-mesenchymal transition. Mol Cancer 13: 68, 2014.

20. Livak KJ and Schmittgen TD: Analysis of relative gene expression data using real-time quantitative PCR and the 2(-Delta Delta C(T)) Method. Methods 25: 402-408, 2001.

21. Li R, Zhang L, Jia L, Duan Y, Li Y, Bao L and Sha N: Long non-coding RNA BANCR promotes proliferation in malignant melanoma by regulating MAPK pathway activation. PLoS One 9: e100893, 2014

22. Li L, Zhang L, Zhang Y and Zhou F: Increased expression of LncRNA BANCR is associated with clinical progression and poor prognosis in gastric cancer. Biomed Pharmacother 72: 109-112, 2015.

23. Su S, Gao J, Wang T, Wang J, Li H and Wang Z: Long non-coding RNA BANCR regulates growth and metastasis and is associated with poor prognosis in retinoblastoma. Tumour Biol 36: 7205-7211, 2015

24. Jiang W, Zhang D, Xu B, Wu Z, Liu S, Zhang L, Tian Y, Han X and Tian D: Long non-coding RNA BANCR promotes proliferation and migration of lung carcinoma via MAPK pathways. Biomed Pharmacother 69: 90-95, 2015.

25. Zheng JH, Viacava Follis A, Kriwacki RW and Moldoveanu T: Discoveries and controversies in BCL-2 protein-mediated apoptosis. FEBS J 283: 2690-2700, 2016.

26. Brentnall M, Rodriguez-Menocal L, De Guevara RL, Cepero E and Boise LH: Caspase-9, caspase-3 and caspase-7 have distinct roles during intrinsic apoptosis. BMC Cell Biol 14: 32, 2013.

27. Guo Q, Zhao Y, Chen J, Hu J, Wang S, Zhang D and Sun Y: BRAF-activated long non-coding RNA contributes to colorectal cancer migration by inducing epithelial-mesenchymal transition. Oncol Lett 8: 869-875, 2014.

28. Galuppo R, Ramaiah D, Ponte OM and Gedaly R: Molecular therapies in hepatocellular carcinoma: What can we target? Dig Dis Sci 59: 1688-1697, 2014.

29. Zhou Q, Lui VW and Yeo W: Targeting the PI3K/Akt/mTOR pathway in hepatocellular carcinoma. Future Oncol 7: 1149-1167, 2011.

30. Bruix J and Sherman M; American Association for the Study of Liver Diseases: Management of hepatocellular carcinoma: An update. Hepatology 53: 1020-1022, 2011.

31. Sicklick JK, Li YX, Jayaraman A, Kannangai R, Qi Y, Vivekanandan P, Ludlow JW, Owzar K, Chen W, Torbenson MS and Diehl AM: Dysregulation of the Hedgehog pathway in human hepatocarcinogenesis. Carcinogenesis 27: 748-757, 2006.

32. Delire B and Stärkel P: The Ras/MAPK pathway and hepatocarcinoma: Pathogenesis and therapeutic implications. Eur J Clin Invest 45: 609-623, 2015.

33. Neuzillet C, Tijeras-Raballand A, de Mestier L, Cros J, Faivre S and Raymond E: MEK in cancer and cancer therapy. Pharmacol Ther 141: 160-171, 2014

34. Wagner EF and Nebreda AR: Signal integration by JNK and p38 MAPK pathways in cancer development. Nat Rev Cancer 9: 537-549, 2009.

35. Gao JJ, Shi ZY, Xia JF, Inagaki Y and Tang W: Sorafenib-based combined molecule targeting in treatment of hepatocellular carcinoma. World J Gastroenterol 21: 12059-12070, 2015.

36. Chen C and Wang G: Mechanisms of hepatocellular carcinoma and challenges and opportunities for molecular targeted therapy. World J Hepatol 7: 1964-1970, 2015. 